\title{
Weak null, necessary defender and necessary detractor players: characterizations of the Banzhaf and the Shapley bisemivalues *
}

\author{
Margarita Domènech, ${ }^{\dagger}$ José Miguel Giménez $z^{\ddagger}$ and María Albina Puente ${ }^{\S}$
}

May 18, 2021

\begin{abstract}
We focus on bicooperative games, a variation of the classic cooperative games and, in particular, on the Banzhaf and the Shapley bisemivalues defined on these games. We consider three special classes of players: weak null, necessary defender and necessary detractor players. By introducing new properties related to this kind of players, we provide new axiomatic characterizations of the Banzhaf and the Shapley bisemivalues giving, in both cases, a set of independent properties that univocally determine them. We also provide a computational procedure to calculate the allocations given by the Shapley bisemivalue in terms of the generalized multilinear extension of the game.
\end{abstract}

Keywords: cooperative game, bicooperative game, Banzhaf bisemivalue, Shapley bisemivalue,

Math. Subj. Class. (2000): 91A12.

\section{Introduction}

This paper focuses on bicooperative games, introduced by Bilbao et al. [5] as a generalization of classical cooperative games, where each player can participate positively to the game, negatively, or do not participate. Formally, ordered pairs of disjoint coalitions of players have to be considered. Thus, each such pair yields a partition of the set of players in three groups: (i) players in the first coalition are defenders of modifying the actual situation and they want to accept a proposal; (ii) players in the second coalition do not agree with it and they will take actions against any change; and (iii) the remaining players are not convinced of the profits of the change, but they do not have intention of objecting to it.

\footnotetext{
*This research project was partially supported by funds from the Spanish Ministry of Science and Innovation grant PID2019-104987GB-I00

${ }^{\dagger}$ Department of Mathematics and Engineering School of Manresa, Technical University of Catalonia, Spain e-mail: margarita.domenech@upc.edu

$¥$ Department of Mathematics and Engineering School of Manresa, Technical University of Catalonia, Spain. e-mail: jose.miguel.gimenez@upc.edu

$\S$ Department of Mathematics and Engineering School of Manresa, Technical University of Catalonia, Spain. e-mail: m.albina.puente@upc.edu (corresponding author)
} 
As in the cooperative case, a central question in bicooperative games is to define a solution concept for a game, that is, a function which assigns to every game a set of real-valued vectors, each one of them represents a payoff distribution among the players. In other words, it is interesting to know how players divide the worth among themselves. The first solution concept to this problem is the Shapley value, introduced and characterized by Bilbao et al. [6] and from now on, called the Shapley bisemivalue.

In 2010 Bilbao et al. [9] analyzed ternary bicooperative games, which are a refinement of the ternary voting games introduced in [21], and defined and axiomatized the Banzhaf power index for these games. In [16], Domènech et al. introduced bisemivalues on bicooperative games in a similar way to the cooperative case given in [19], and generalized this power index by introducing the Banzhaf bisemivalue on bicooperative games as a particular case of a subfamily of bisemivalues, called $(p, q)$-bisemivalues. For each one of them, the weighting coefficients depend on two parameters $p, q \in[0,1]$. These bisemivalues are suited for the study of bicooperative games where players show two different tendencies to form coalitions. These tendencies are defined for all players by parameters $p$ and $q$. The particular case $p=q=1 / 3$ gives rise the Banzhaf bisemivalue on bicooperative games.

The Banzhaf bisemivalue of a player is the average of the contributions of this player to all pair of coalitions either he/she joins or he/she leaves. However, the Shapley bisemivalue of a player is a weighted average of those contributions but, now, the weights depend on the size of the coalitions. In a recent paper, Domènech et al. [17] investigated the conditions for the coefficients of the bisemivalues, necessary and / or sufficient, in order to satisfy some standard properties, considered for values on cooperative games (see, e.g., [10, 15, 21]). For more information about solution concepts on bicooperative games we refer the reader to [7] and [8].

Since Shapley [33] initiated the value theory for cooperative games, several axiomatic characterizations of the Shapley and the Banzhaf [4] values of cooperative games have been proposed in literature. The Shapley value applies without restrictions and provides, for every game, a single payoff vector to the players. The restriction of the value to simple games gives rise to the Shapley-Shubik power index [34], that was axiomatized by Dubey [18] introducing the transfer property. As a sort of reaction, Banzhaf [4] proposed a different power index (see also Coleman [13], and even Penrose [32]), that Owen [30] extended to a dummy-independent and somehow "normalized" Banzhaf value for all cooperative games. A nice almost common characterization of the Shapley and Banzhaf values would be given by Feltkamp [20], and a sound interpretative and comparative analysis has been carried out by Laruelle and Valenciano [27]. See also Lehrer [28] and Owen [31]. Interesting contributed chapters related to the Shapley value can be found in [1].

The nullifying players were defined in [14] and, more recently in [35], Van den Brink provided a characterization of the Equal Division Solution using, among others, the property of nullifying agents get nothing. As this property does not hold for the Banzhaf and the Shapley value, Alonso-Meijide et al. [3], proposed two new properties related to these players in order to characterize both classical values. In fact, they obtained new characterizations of the Banzhaf and Shapley values on cooperative games considering null, nullifying and necessary players. They used the additivity property, a classical property that has been included in many characterizations of both values, and introduced the property of nullifying agents pay for the mean and the property of necessary agents get the mean for the Banzhaf value; and the 
property of nullifying agents pay for the weighted mean and the property of necessary agents get the weighted mean for the Shapley value. All these new properties suggest payoffs for these players.

Later on, the concept of dummifying player is introduced in [12] to illustrate the difference between the Shapley value, the Equal Division Solution and the Equal Surplus value. In [2], new axiomatic characterizations of the Shapley and the Banzhaf values using properties involving either nullifying players or dummifying players are also provided.

Another problem highly considered in cooperative games has been the computation of values, usually in terms of the multilinear extension of the game, introduced by Owen [29]. Thus, Owen [29] refers to the Shapley value [33] and Owen [30] to the Banzhaf value [4]. Following this schema, Domènech et al. in [16], defined a multilinear extension for each bicooperative game and gave a computational procedure to calculate $(p, q)$-bisemivalues in general, and the Banzhaf bisemivalue in particular. Moreover, in [17] a computational procedure to calculate bisemivalues in terms of the multilinear extension of the game was also given.

The aim of this paper is to provide new axiomatic characterizations of the Banzhaf and Shapley bisemivalues on bicooperative games in a similar way to given in [3] for cooperative games. In other words, we "extend" these results to the bicooperative case and provide new axiomatic characterizations of these two bisemivalues. To do this, we define the concept of three special types of players: weak null player, necessary defender player and necessary detractor player; and we also introduce new non-standard properties that propose reasonable payoffs for these players. Then, by considering these properties and some classical properties as linearity, efficiency and symmetry, we obtain parallel axiomatic characterizations of these two bisemivalues. In the case of the Banzhaf value, two new axiomatic characterizations are given: the second one is obtained by just replacing the property of necessary defender player with the property of necessary detractor player in the first axiomatic characterization.

The organization of the paper is as follows. In Section 2, we include a minimum of preliminaries that refers to cooperative games and bicooperative games. In Section 3 and 4 we focus on the Banzhaf and Shapley bisemivalues respectively, giving systems of independent properties to characterize them. Finally, Section 5 is devoted to give a computational procedure to calculate the Shapley bisemivalue in terms of the generalized multilinear extension of the game.

\section{Preliminaries}

\subsection{Cooperative games and bicooperative games}

Let $N$ be a finite set of players and $2^{N}$ be the set of its coalitions (subsets of $N$ ). A cooperative game on $N$ is a function $v: 2^{N} \rightarrow \mathbb{R}$, that assigns a real number $v(S)$ to each coalition $S \subseteq N$, with $v(\emptyset)=0$. A game $v$ is monotonic if $v(S) \leq v(T)$ whenever $S \subseteq T \subseteq N$ and simple if, moreover, $v(S)=0$ or 1 for every $S \subseteq N$. Endowed with the natural operations for real-valued functions, i.e. $v+v^{\prime}$ and $\lambda v$ for all $\lambda \in \mathbb{R}$, the set $\mathcal{G}_{N}$ of all cooperative games in $N$ becomes a vector space.

Let $(N, v)$ be a cooperative game; we say that

- $i \in N$ is a dummy player in $v$ if $v(S \cup\{i\})=v(S)+(\{i\})$ for all $S \subseteq N \backslash\{i\}$, 
- $i \in N$ is a null player in $v$ if $v(S \cup\{i\})=v(S)$ for all $S \subseteq N \backslash\{i\}$,

- $i \in N$ is a necessary player in $v$ if $v(S)=0$ for all $S \subseteq N \backslash\{i\}$,

- $i \in N$ is a nullifying player in $v$ if $v(S)=0$ when $i \in S$,

- $i \in N$ is a dummifying player in $v$ if $v(S)=\sum_{i \in S} v(\{i\})$ for all $S \subseteq N$ with $i \in S$.

A dummy player contributes his/her individual worth to each coalition he/she joins. A null player is one that contributes nothing to each coalition he/she joins. A necessary player nullifies the worth of any coalition to which he/she does not belong. A nullifying player is one such that all the coalitions containing him/her have zero worth and a dummifying player is one such that each coalition containing him/her has a worth equal to the sum of the individual worths of its members.

If $(N, v)$ is a simple game; we say that $i \in N$ is a veto player in $v$ if $v(N \backslash\{i\})=0$. Notice that the concept of necessary player is an extension to cooperative games of the concept of veto player in a simple game.

By a value on $\mathcal{G}_{N}$ we will mean a map $f: \mathcal{G}_{N} \rightarrow \mathbb{R}^{n}$, that assigns to every game $v$ a vector $f[v]$ with components $f_{i}[v]$ for all $i \in N$. In particular, the Banzhaf value $\beta$, is given by

$$
\beta_{i}[v]=\frac{1}{2^{n-1}} \sum_{S \subseteq N \backslash\{i\}}[v(S \cup\{i\})-v(S)] \text { for all } i \in N \text { and for all } v \in \mathcal{G}_{N},
$$

and the Shapley value $\varphi$ by

$$
\varphi_{i}[v]=\frac{1}{n} \sum_{S \subseteq N \backslash\{i\}} \frac{1}{\left(\begin{array}{c}
n-1 \\
s
\end{array}\right)}[v(S \cup\{i\})-v(S)] \text { for all } i \in N \text { and for all } v \in \mathcal{G}_{N} .
$$

Notice that the $\beta_{i}[v]$ is the average of the contributions of player $i$ to all coalitions to which it does not belong and $\varphi_{i}[v]$ is a weighted average of those contributions, where now the weights depend on the size of coalitions $S$.

Properties related to null players, necessary players and nullifying players are used in [2] and [3] to provide characterizations of the Shapley and the Banzhaf values.

The multilinear extension ${ }^{1}$ [29] of a game $v \in \mathcal{G}_{N}$ is the real-valued function defined on $\mathbb{R}^{n}$ by

$$
f_{v}\left(x_{1}, x_{2}, \ldots, x_{n}\right)=\sum_{S \subseteq N} \prod_{i \in S} x_{i} \prod_{j \in N \backslash S}\left(1-x_{j}\right) v(S),
$$

where $0 \leq x_{i} \leq 1$ for $i=1,2, \ldots, n$.

As it is well known, the Shapley and the Banzhaf values can be obtained by the multilinear extension of the game (see [29] and [30]).

Let $N$ be a finite set of players and $3^{N}=\{(S, T): S, T \subseteq N, S \cap T=\emptyset\}$ be the set of all ordered pairs of disjoint coalitions. Grabisch and Labreuche [26] proposed a relation in $3^{N}$ given by

$$
(A, B) \sqsubseteq(C, D) \Leftrightarrow A \subseteq C, B \supseteq D .
$$

\footnotetext{
${ }^{1}$ The term "multilinear" means that, for each $i \in N$, the function is linear in $x_{i}$, that is, of the form $f_{v}\left(x_{1}, x_{2}, \ldots, x_{n}\right)=g_{i}\left(x_{1}, x_{2}, \ldots, x_{i}, \ldots, x_{n}\right) x_{i}+h_{i}\left(x_{1}, x_{2}, \ldots, \hat{x}_{i}, \ldots, x_{n}\right)$.
} 
The set $\left(3^{N}, \sqsubseteq\right)$ is a partially ordered set (or poset) and moreover, it is a finite distributive lattice. Two pairs $(A, B)$ and $(C, D)$ are comparable if $(A, B) \sqsubseteq(C, D)$ or $(C, D) \sqsubseteq(A, B)$; otherwise, $(A, B)$ and $(C, D)$ are incomparable. A chain of $3^{N}$ is an induced subposet of $3^{N}$ in which any two elements are comparable. Thus, the rank function

$$
\rho: 3^{N} \rightarrow\{0,1,2, \ldots, 2 n\}
$$

is defined by $\rho[(\emptyset, N)]=0$ and $\rho[(S, T)]=\rho[(A, B)]+1$ if $(S, T)$ covers $(A, B)$, that is, if $(A, B) \sqsubset(S, T)$ and there exists no $(H, J) \in 3^{N}$ such that $(A, B) \sqsubset(H, J) \sqsubset(S, T)$.

Following [5], a bicooperative game on $N$ is a function $b: 3^{N} \rightarrow \mathbb{R}$, that assigns a real number $b(S, T)$ to each pair of coalitions $(S, T) \in 3^{N}$, with $b(\emptyset, \emptyset)=0$. For each $(S, T) \in 3^{N}$, the worth $b(S, T)$ can be interpreted as the maximal gain (if $b(S, T)>0$ ) or the minimal loss (if $b(S, T)<0)$ that the members of coalition $S$ can achieve when they decide to play together against the members of $T$ and the members of $N \backslash(S \cup T)$ do not take part. Then $b(\emptyset, N)$ is the cost obtained when all players are against the change and $b(N, \emptyset)$ is the maximal gain obtained when all players want to change the initial situation.

A bicooperative game is monotonic if $b(S, T) \leq b\left(S^{\prime}, T^{\prime}\right)$ whenever $(S, T) \sqsubseteq\left(S^{\prime}, T^{\prime}\right)$. Felsenthal and Machover [21] generalize the standard voting games by recognizing abstention as an option alongside yes and no votes and introduce the ternary voting games as a map $u: 3^{N} \rightarrow$ $\{-1,1\}$ satisfying the following conditions: monotonicity, $u(N, \emptyset)=1$ and $u(\emptyset, N)=-1$. A negative outcome, -1 , is interpreted as a defeat and a positive outcome, 1 , as a victory, the passing of a bill. The proposal of Felsenthal and Machover could be refined by introducing a third output for $\mathrm{u}$, which is 0 , and represents the 'no decision' situation. Thus, Bilbao et al [9] considered that a bicooperative game $b$ is ternary if it is monotonic and, moreover $b(S, T) \in\{-1,0,1\}$ for all $(S, T) \in 3^{N}$. Several works by Freixas [22], [23] and Freixas and Zwicker [25] have been devoted to the study of voting systems with several ordered levels of approval in the input and in the output. In their model, the abstention is a level of input approval intermediate between yes and no votes.

A player $i \in N$ is a dummy in $b$ if $b(S \cup\{i\}, T)=b(S, T)+b(\{i\}, \emptyset)$ and $b(S, T \cup\{i\})=$ $b(S, T)+b(\emptyset,\{i\})$ for all $(S, T) \in 3^{N \backslash\{i\}}$, and null in $b$ if, moreover, $b(\{i\}, \emptyset)=b(\emptyset,\{i\})=0$. Notice that if $i$ is a null player, then $b(S \cup\{i\}, T)=b(S, T \cup\{i\})$.

Two players $i, j \in N$ are symmetric in $b$ if $b(S \cup\{i\}, T)=b(S \cup\{j\}, T), b(S, T \cup\{i\})=$ $b(S, T \cup\{j\})$ and $b(S \cup\{i\}, T \cup\{j\})=b(S \cup\{j\}, T \cup\{i\})$ for all $(S, T) \in 3^{N \backslash\{i, j\}}$.

Endowed with the natural operations for real-valued functions, the set of all bicooperative games on $N$ is a vector space that we will denote by $\mathcal{B} \mathcal{G}_{N}$. For every $(S, T) \in 3^{N}$ such that $(S, T) \neq(\emptyset, \emptyset)$, the identity game $\delta_{(S, T)}$ is defined by $\delta_{(S, T)}(A, B)=1$ if $(A, B)=(S, T)$ and $\delta_{(S, T)}(A, B)=0$ otherwise. It is easily checked that the set of all identity games are a basis for $\mathcal{B} \mathcal{G}_{N}$, so that $\operatorname{dim}\left(\mathcal{B G}_{N}\right)=3^{n}-1$ if $n=|N|$.

By a value on $\mathcal{B G}_{N}$ we will mean a map $g: \mathcal{B} \mathcal{G}_{N} \rightarrow \mathbb{R}^{n}$, that assigns to every game $b$ a vector $g[b]$ with components $g_{i}[b]$ for all $i \in N$.

\subsection{Bisemivalues}

Following [16], a bisemivalue on bicooperative games is a map $\psi: \mathcal{B} \mathcal{G}_{N} \rightarrow \mathbb{R}^{n}$ that satisfies: 
(i) linearity: $\psi\left[\alpha b+\beta b^{\prime}\right]=\alpha \psi[b]+\beta \psi\left[b^{\prime}\right]$, for all $b, b^{\prime} \in \mathcal{G} \mathcal{B}_{N}$ and $\alpha, \beta \in \mathbb{R}$;

(ii) symmetry: $\psi_{\pi i}[\pi b]=\psi_{i}[b]$ for all permutation $\pi$ over $N, i \in N$, and $b \in \mathcal{B G}_{N}$, where $\pi b(\pi S, \pi T)=b(S, T)$ and $\pi S=\{\pi i: i \in S\}$

(iii) positivity: if $b$ is monotonic, then $\psi[b] \geq 0$;

(iv) dummy player property: if $i \in N$ is a dummy in game $b$ then, $\psi_{i}[b]=b(\{i\}, \emptyset)-$ $b(\emptyset,\{i\})$.

Remark 2.1 Notice that the property of symmetry can be written as follows: if $i, j \in N$ are symmetric in $b$, then $\psi_{i}[b]=\psi_{j}[b]$.

Domènech et al. in [16], provided an interesting characterization of the bisemivalues by weighting coefficients in a similar way to the cooperative case given in [19], as follows.

Theorem 2.2 (Domènech et al, [16]) A value $\psi$ on $\mathcal{B G}_{N}$ is a bisemivalue if and only if there exist two collections of real numbers $p_{s, t}$ and $q_{s, t}, s, t=0, .1, \ldots, n-1$, satisfying:

$$
\begin{aligned}
& p_{s, t} \geq 0, q_{s, t} \geq 0, \\
& \sum_{s=0}^{n-1}\left(\begin{array}{c}
n-1 \\
s
\end{array}\right)\left[\sum_{t=0}^{n-s-1}\left(\begin{array}{c}
n-s-1 \\
t
\end{array}\right) p_{s, t}\right]=1, \\
& \sum_{t=0}^{n-1}\left(\begin{array}{c}
n-1 \\
t
\end{array}\right)\left[\sum_{s=0}^{n-t-1}\left(\begin{array}{c}
n-t-1 \\
s
\end{array}\right) q_{s, t}\right]=1,
\end{aligned}
$$

such that

$$
\psi_{i}[b]=\sum_{(S, T) \in 3^{N \backslash\{i\}}}\left[p_{s, t}(b(S \cup\{i\}, T)-b(S, T))+q_{s, t}(b(S, T)-b(S, T \cup\{i\}))\right]
$$

for all $i \in N$ and all $b \in \mathcal{B}_{\mathcal{G}_{N}}$, where $s=|S|$ and $t=|T|$.

Remark 2.3 (a) The payoff that a bisemivalue allocates to every player in any game is a weighted sum of his/her marginal contributions $b(S \cup\{i\}, T)-b(S, T)$ whenever $i$ joins coalition $S \subseteq N \backslash i$ and his/her marginal contributions $b(S, T)-b(S, T \cup\{i\})$ whenever $i$ leaves coalition $T \cup\{i\}$; where $p_{s, t}$ is the probability that player $i$ joins $S$ in presence of the players in $T$ and $q_{s, t}$ is the probability that player $i$ leaves $T \cup\{i\}$ in presence of the players in $S$, provided that all the coalitions of a common size have the same probability of being joined and left.

(b) Among bisemivalues, we find the Shapley bisemivalue [6] $\varphi$ and the Banzhaf bisemivalue [16] $\beta$, whose weighting coefficients $p_{s, t}$ and $q_{s, t}$ are defined in Section 3 and 4 respectively.

Taking into account that the identity games form a basis on $\mathcal{B G}_{N}$, it is interesting to know the allocations that a player gets in these games when a bisemivalue is applied. 
Lemma 2.4 ( Domènech et al. [17]) Let $\psi$ be a bisemivalue on $\mathcal{B G}_{N}$. The allocations to a player $i \in N$ in the identity game $\delta_{(A, B)}$ are given by

$$
\psi_{i}\left[\boldsymbol{\delta}_{(A, B)}\right]= \begin{cases}p_{a-1, b}, & \text { if } i \in A, \\ -q_{a, b-1}, & \text { if } i \in B, \\ q_{a, b}-p_{a, b}, & \text { if } i \in N \backslash(A \cup B) .\end{cases}
$$

\section{The Banzhaf bisemivalue}

In [9], the Banzhaf power index for ternary bicooperative games is characterized as the unique power index satisfying null player, total swings (or total power), transfer, simple additivity and structural properties. This section is devoted to the discussion of common properties for the Banzhaf bisemivalue in order to rich two axiomatic characterizations of it among the values on the set of all bicooperative games. A part from the standard properties considered in value theory, new two additional non-standard properties will be considered. First of all, let us remind you of the definition of the Banzhaf bisemivalue [16].

Definition 3.1 The Banzhaf bisemivalue $\beta$ is defined as

$$
\beta_{i}[b]=\frac{1}{3^{n-1}} \sum_{(S, T) \in 3^{N \backslash\{i\}}}[b(S \cup\{i\}, T)-b(S, T \cup\{i\})] \text {, for all } i \in N \text { and } b \in \mathcal{B} \mathcal{G}_{N}
$$

It is easy to prove that $\beta$ is the only bisemivalue with coefficients $p_{s, t}, q_{s, t}$ constant, that is, they do not depend on the size of coalitions $(S, T)$.

As we have said before, a player $i \in N$ is null in $b$ iff he/she is a dummy and moreover, $b(\{i\}, \emptyset)=b(\emptyset,\{i\})=0$. It is clear that if $i$ is a null player, then $b(S \cup\{i\}, T)=b(S, T \cup\{i\})$, but the contrary is not true in general. This fact leads us to consider the following definition.

Definition 3.2 A player $i \in N$ is a weak null player in $b$ iff $b(S \cup\{i\}, T)=b(S, T \cup\{i\})$ for all $(S, T) \in 3^{N \backslash\{i\}}$.

The following property states that a value must allocate zero to any weak null player of any bicooperative game.

Definition 3.3 A value $g$ in $\mathcal{B} \mathcal{G}_{N}$ satisfies the weak null property if for all $b \in \mathcal{B} \mathcal{G}_{N}$ and for all weak null player $i \in N, g_{i}[b]=0$.

Remark 3.4 Notice that this property does not hold for all bisemivalue in general. In fact, it is easy to prove that a bisemivalue $\psi$ satisfies the weak null player property iff $p_{s, t}=q_{s, t}$, for all $s, t=0,1, \ldots, n-1$.

In order to obtain the axiomatic characterization of the Banzhaf bisemivalue, we need to introduce the concepts of necessary defender player and necessary detractor player. 
Definition 3.5 (i) A player $i \in N$ is a necessary defender player in $b$ iff $b(S, T)=0$, for all $S \subseteq N \backslash\{i\}$.

(ii) A player $i \in N$ is a necessary detractor player in $b$ iff $b(S, T)=0$, for all $T \subseteq N \backslash\{i\}$.

We will give new properties that determine the payoff for this special players. These properties adapt to bicooperative games similar properties introduced in [3] when characterizing the Shapley and the Banzhaf values for cooperative games.

Remark 3.6 (a) Given $(A, B) \neq(\emptyset, \emptyset)$, notice that players in $A$ are necessary defender players; players in $B$ are necessary detractor players and players in $N \backslash(A \cup B)$ are weak null, all of them on the identity game $\delta_{(A, B)}$.

(b) The concept of necessary defender player can be considered as the extension to bicooperative games of the concept of veto player.

In the case of a necessary defender player $i \in N$, a pair of coalitions $(S, T)$ receives nothing if $i \notin S$ in a similar way to the cooperative case. That is, a proposal can not prosper without $\mathrm{him} / \mathrm{her}$. Then, this kind of players should get the greatest payoff. The following property proposes that they obtain the average worth over all coalitions $(S, T) \in 3^{N}$.

Definition 3.7 A value $g$ on $\mathcal{B} \mathcal{G}_{N}$ satisfies the property of necessary defender player if for all $b \in \mathcal{B} G_{N}$ and for all necessary defender player $i \in N$, it holds that

$$
g_{i}[b]=\frac{1}{3^{n-1}} \sum_{(S, T) \in 3^{N}} b(S, T) .
$$

In the case of a necessary detractor player $i \in N$, a pair of coalitions $(S, T)$ receives nothing if $i \in S \cup N \backslash(S \cup T)$ in a similar way to nullifying players in cooperative games. That is, all the coalitions $(S, T)$, with $i \notin T$, have zero worth. The following property states that a value on $\mathcal{B} \mathcal{G}_{N}$ must allocate to any detractor player of any bicooperative game minus the average worth over all coalitions $(S, T) \in 3^{N}$.

Definition 3.8 A value $g$ on $\mathcal{B} \mathcal{G}_{N}$ satisfies the property of necessary detractor player if for all $b \in \mathcal{B} \mathcal{G}_{N}$ and for all necessary defender player $i \in N$, it holds that

$$
g_{i}[b]=-\frac{1}{3^{n-1}} \sum_{(S, T) \in 3^{N}} b(S, T)
$$

Theorem 3.9 (First axiomatic caracterization of the Banzhaf bisemivalue) The Banzhaf bisemivalue is the only value on $\mathcal{B} \mathcal{G}_{N}$ that satisfies the properties of linearity, weak null player and necessary defender player.

Proof (Existence) It is straightforward that the Banzhaf bisemivalue satisfies linearity and the weak null player property.

If $i \in N$ is a necessary defender player in $b$ then,

$$
\sum_{(S, T) \in 3^{N}} b(S, T)=\sum_{(S, T) \in 3^{N} \backslash\{i\}}[b(S, T)+b(S \cup\{i\}, T)+b(S, T \cup\{i\})]=\sum_{(S, T) \in 3^{N \backslash\{i\}}} b(S \cup\{i\}, T)
$$


and then,

$$
\begin{aligned}
\beta_{i}[b] & =\frac{1}{3^{n-1}} \sum_{(S, T) \in 3^{N \backslash\{i\}}}[b(S \cup\{i\}, T)-b(S, T \cup\{i\})] \\
& =\frac{1}{3^{n-1}} \sum_{(S, T) \in 3^{N \backslash\{i\}}} b(S \cup\{i\}, T)=\frac{1}{3^{n-1}} \sum_{(S, T) \in 3^{N}} b(S, T) .
\end{aligned}
$$

Thus, $\beta$ also satisfies the property of necessary defender player.

(Uniqueness) Let $g$ be a value on $\mathcal{B G}_{N}$ that satisfies the started properties. We will show that $g$ is uniquely determined on all $b \in \mathcal{B} \mathcal{G}_{N}$; so that it must coincide with $\beta$. Using linearity, we need only to prove that $g$ is uniquely determined on each identity game $\delta_{(A, B)}$.

Given $(A, B) \in 3^{N}$ such that $(A, B) \neq(\emptyset, \emptyset)$, from Lemma 2.4 the allocations to a player $i \in N$ in the identity game $\delta_{(A, B)}$ given for the particular case of the Banzhaf bisemivalue are

$$
\beta_{i}\left[\boldsymbol{\delta}_{(A, B)}\right]=\left\{\begin{array}{cl}
\frac{1}{3^{n-1}}, & \text { if } i \in A, \\
-\frac{1}{3^{n-1}}, & \text { if } i \in B, \\
0, & \text { if } i \in N \backslash(A \cup B) .
\end{array}\right.
$$

We will distinguish 3 cases.

(i) If $i \in N \backslash(A \cup B)$ then, $i$ is a weak null player in $\delta_{(A, B)}$ and, applying the property of weak null player, it follows that

$$
g_{i}\left[\boldsymbol{\delta}_{(A, B)}\right]=0=\beta_{i}\left[\boldsymbol{\delta}_{(A, B)}\right] .
$$

(ii) If $i \in A$ then, $i$ is a necessary defender player in $\delta_{(A, B)}$. By applying the property of necessary defender player, we get:

$$
g_{i}\left[\delta_{(A, B)}\right]=\frac{1}{3^{n-1}} \sum_{(S, T) \in 3^{N}} \delta_{(A, B)}(S, T)=\frac{1}{3^{n-1}}=\beta_{i}\left[\delta_{(A, B)}\right] .
$$

(iii) If $i \in B$, we can write $B=B^{*} \cup\{i\}$, where, $B^{*} \subseteq N \backslash\{i\}$. Consider the game

$$
b^{*}=\delta_{\left(A \cup\{i\}, B^{*}\right)}+\delta_{\left(A, B^{*} \cup\{i\}\right)}
$$

It is easy to check that $i$ is a necessary defender player in $\delta_{\left(A \cup\{i\}, B^{*}\right)}$ and then,

$$
g_{i}\left[\boldsymbol{\delta}_{\left(A \cup\{i\}, B^{*}\right)}\right]=\beta_{i}\left[\boldsymbol{\delta}_{\left(A \cup\{i\}, B^{*}\right)}\right]=\frac{1}{3^{n-1}}
$$

Moreover, $i$ is a weak null in $b^{*}$ and, by the weak null property, we have

$$
g_{i}\left[b^{*}\right]=0
$$

By linearity, it follows that

$$
0=g_{i}\left[b^{*}\right]=\frac{1}{3^{n-1}}+g_{i}\left[\boldsymbol{\delta}_{\left(A, B^{*} \cup\{i\}\right)}\right]
$$


and thus, we get:

$$
g_{i}\left[\boldsymbol{\delta}_{(A, B)}\right]=g_{i}\left[\boldsymbol{\delta}_{\left(A, B^{*} \cup\{i\}\right)}\right]=-\frac{1}{3^{n-1}}=\beta_{i}\left[\boldsymbol{\delta}_{(A, B)}\right]
$$

Proposition 3.10 (Logical independence of the system used in Theorem 3.9) We will assume that a player set $N$ (with $n=|N| \geq 2$ ) is given.

We will abbreviate the properties as follows: $L I=$ linearity, $W N P=$ weak null player property and $D E F=$ necessary defender player property.

\section{LI is logically independent of WNP and DEF}

For $k \neq 0$, we define a value $g$ for all $\mathcal{B G}_{N}$ as follows:

$$
g_{i}[b]=\left\{\begin{array}{cl}
\beta_{i}[b], & \text { if } i \text { is either a weak null player or a necessary defender player in } b \\
k & \text { otherwise. }
\end{array}\right.
$$

This value satisfies WNP and DEF. However, it fails to satisfy LI.

2. WNP logically independent of LI, and DEF.

We define a value $g$ for all $\mathcal{B}_{\mathcal{G}_{N}}$ as follows:

$$
g_{i}[b]=\frac{1}{3^{n-1}} \sum_{(S, T) \in 3^{N}} b(S, T), \text { for all } i \in N .
$$

This value satisfies LI and DEF. However, it fails to satisfy WNP

3. DEF is logically independent of LI, and WNP.

For $k \neq 1$, we define a value $g$ for all $\mathcal{B} \mathcal{G}_{N}$ as follows:

$$
g_{i}[b]=k \beta_{i}[b], \text { for all } i \in N
$$

This value satisfies LI and WNP. However, it fails to satisfy DEF.

As we will see in the following theorem, the second axiomatic characterization of the Banzhaf bisemivalue is obtained by just replacing the property of necessary defender player with the property of necessary detractor player in the first characterization.

Theorem 3.11 (Second axiomatic caracterization of the Banzhaf bisemivalue) The Banzhaf bisemivalue is the only value on $\mathcal{B G}_{N}$ that satisfies the properties of linearity, weak null player and necessary detractor player. 
Proof (Existence) It is straightforward that the Banzhaf bisemivalue satisfies linearity and the weak null player property.

If $i \in N$ is a necessary detractor player in $b$ then,

$$
\sum_{(S, T) \in 3^{N}} b(S, T)=\sum_{(S, T) \in 3^{N \backslash\{i\}}}[b(S, T)+b(S \cup\{i\}, T)+b(S, T \cup\{i\})]=\sum_{(S, T) \in 3^{N \backslash\{i\}}} b(S, T \cup\{i\})
$$

and then,

$$
\begin{aligned}
\beta_{i}[b] & =\frac{1}{3^{n-1}} \sum_{(S, T) \in 3^{N \backslash\{i\}}}[b(S \cup\{i\}, T)-b(S, T \cup\{i\})] \\
& =-\frac{1}{3^{n-1}} \sum_{(S, T) \in 3^{N \backslash\{i\}}} b(S, T \cup\{i\})=-\frac{1}{3^{n-1}} \sum_{(S, T) \in 3^{N}} b(S, T) .
\end{aligned}
$$

Thus, $\beta$ also satisfies the property of necessary detractor player.

(Uniqueness) Let $g$ be a value on $\mathcal{B G}_{N}$ that satisfies the started properties. We will show that $g$ is uniquely determined on all $b \in \mathcal{B G}_{N}$; so that it must coincide with $\beta$. Using linearity, we need only to prove that $g$ is uniquely determined on each identity game $\delta_{(A, B)}$. As in the previous theorem, we will take into account the allocations given in Lemma 2.4 for the Banzhaf bisemivalue.

Given $(A, B) \in 3^{N}$ such that $(A, B) \neq(\emptyset, \emptyset)$, we will distinguish 3 cases.

(i) If $i \in N \backslash(A \cup B)$ then, $i$ is a weak null player in $\delta_{(A, B)}$ and, applying the property of weak null player, it follows that

$$
g_{i}\left[\boldsymbol{\delta}_{(A, B)}\right]=0=\beta_{i}\left[\boldsymbol{\delta}_{(A, B)}\right] .
$$

(ii) If $i \in B$ then, $i$ is a necessary detractor player in $\delta_{(A, B)}$ and the property of necessary detractor player imply that

$$
g_{i}\left[\boldsymbol{\delta}_{(A, B)}\right]=-\frac{1}{3^{n-1}} \sum_{(S, T) \in 3^{N}} \delta_{(A, B)}(S, T)=-\frac{1}{3^{n-1}}=\beta_{i}\left[\boldsymbol{\delta}_{(A, B)}\right] .
$$

(iii) If $i \in A$, we can write $A=A^{*} \cup\{i\}$, where, $A^{*} \subseteq N \backslash\{i\}$. Consider the game

$$
b^{* *}=\delta_{\left(A^{*} \cup\{i\}, B\right)}+\delta_{\left(A^{*}, B \cup\{i\}\right)}
$$

It is easy to check that $i$ is a necessary detractor player in $\delta_{\left(A^{*}, B \cup\{i\}\right)}$ and then,

$$
g_{i}\left[\boldsymbol{\delta}_{\left(A^{*}, B \cup\{i\}\right)}\right]=\beta_{i}\left[\boldsymbol{\delta}_{\left(A^{*}, B \cup\{i\}\right)}\right]=-\frac{1}{3^{n-1}}
$$

Moreover, $i$ is a weak null in $b^{* *}$ and, by the weak null property, we have

$$
g_{i}\left[b^{* *}\right]=0
$$


By linearity, it follows that

$$
0=g_{i}\left[b^{* *}\right]=g_{i}\left[\delta_{\left(A^{*} \cup\{i\}, B\right)}\right]-\frac{1}{3^{n-1}}
$$

and thus,

$$
g_{i}\left[\boldsymbol{\delta}_{(A, B)}\right]=g_{i}\left[\boldsymbol{\delta}_{\left(A^{*} \cup\{i\}, B\right)}\right]=\frac{1}{3^{n-1}}=\beta_{i}\left[\boldsymbol{\delta}_{(A, B)}\right]
$$

Proposition 3.12 (Logical independence of the system used in Theorem 3.11) We will assume that a player set $N$ (with $n=|N| \geq 2$ ) is given.

We will abbreviate the properties as follows: $L I=$ linearity, $W N P=$ weak null player property and DET = necessary detractor player property.

\section{LI is logically independent of WNP and DET}

For $k \neq 0$, we define a value $g$ for all $\mathcal{B G}_{N}$ as follows:

$$
g_{i}[b]=\left\{\begin{array}{cl}
\beta_{i}[b], & \text { if } i \text { is either a weak null player or a necessary detractor player in } b \\
k & \text { otherwise. }
\end{array}\right.
$$

This value satisfies WNP and DET. However, it fails to satisfy LI.

2. WNP logically independent of LI, and DET.

We define a value $g$ for all $\mathcal{B}_{N}$ as follows:

$$
g_{i}[b]=-\frac{1}{3^{n-1}} \sum_{(S, T) \in 3^{N}} b(S, T), \text { for all } i \in N .
$$

This value satisfies LI and DET. However, it fails to satisfy WNP

\section{DET is logically independent of LI, and WNP.}

For $k \neq 1$, we define a value $g$ for all $\mathcal{B G}_{N}$ as follows:

$$
g_{i}[b]=k \beta_{i}[b], \text { for all } i \in N
$$

This value satisfies LI and WNP. However, it fails to satisfy DET.

Remark 3.13 In Theorem 3.11 we have obtained a new characterization of the Banzhaf bisemivalue by just replacing in Theorem 3.9 the property of necessary defender player with the property of necessary detractor player. However, this new property can not substitute weak null player property. That is, linearity, necessary defender player property and necessary detractor player property do not characterize the Banzhaf bisemivalue.

For instance, we define the value $g \in \mathcal{B} \mathcal{G}_{N}$ as follows: 
(a) If $n=2$ then,

$$
\begin{aligned}
& g_{1}[b]=\beta_{1}[b]-[b(\{2\}, \emptyset)-b(\emptyset,\{2\})] \\
& g_{2}[b]=\beta_{2}[b]-[b(\{1\}, \emptyset)-b(\emptyset,\{1\})] .
\end{aligned}
$$

(b) Otherwise, that is, if $n>2$, we define the value $g_{i}[b]=\beta_{i}[b]$, for all $i \in N$.

This value satisfies linearity, necessary defender property and necessary detractor property and it is not the Banzhaf value.

\section{The Shapley bisemivalue}

The Shapley bisemivalue was characterized by Bilbao et al [6] as the only value on $\mathcal{B G}_{N}$ satisfying efficiency -in the sense that its total power for every $b \in \mathcal{B} \mathcal{G}_{N}$ is $\sum_{i \in N} \varphi_{i}[b]=$ $b(N, \emptyset)-b(\emptyset, N)-$, linearity, dummy player property, symmetry and a structural axiom.

In this section, we provide a new axiomatic characterization of this bisemivalue by combining classical properties as linearity, symmetry and efficiency, with two new non-stardard properties related to defender and necessary detractor players.

First of all, we remind you of the definition of the Shapley bisemivalue.

Definition 4.1 The Shapley bisemivalue $\varphi$ is defined as

$\varphi_{i}[b]=\sum_{(S, T) \in 3^{N \backslash\{i\}}}\left[p_{s, t}(b(S \cup\{i\}, T)-b(S, T))+q_{s, t}(b(S, T)-b(S, T \cup\{i\}))\right]$, for all $i \in N$ and for all $b \in \mathcal{B G}_{N}$, where

$$
p_{s, t}=\frac{(n+s-t) !(n+t-s-1) !}{(2 n) !} 2^{n-s-t} \text { and } q_{s, t}=\frac{(n+t-s) !(n+s-t-1) !}{(2 n) !} 2^{n-s-t},
$$

for all $s, t=0,1, \ldots, n-1$.

Remark 4.2 Following the results obtained in [6], we have:

(a) The number of maximal chains of $3^{N}$ is $(2 n) ! / 2^{n}$.

(b) For all $(A, B) \in 3^{N}$, the number of maximal chains of the sublattice $[(\emptyset, N),(A, B)]$ is $(n+a-b) ! / 2^{a}$, where $a=|A|$ and $b=|B|$.

(c) Let $(A, B),(C, D) \in 3^{N}$ with $(A, B) \sqsubseteq(C, D)$. The number of maximal chains in the sublattice $[(A, B),(C, D)]$ is equal to the number of maximal chains in the sublattice $[(D, C),(B, A)]$.

From now on, we will denote by $c[(A, B),(C, D)]$ the number of maximal chains in the sublattice $[(A, B),(C, D)]$. 
Remark 4.3 Remember that $p_{s, t}$ can be interpreted as the probability that player $i$ joins $S$ in presence of the players in $T$ and $q_{s, t}$ as the probability that player $i$ leaves $T \cup\{i\}$ in presence of the players in $S$, provided that all the coalitions of a common size have the same probability of being joined and left. Assuming that all sequential orders of chains have the same probability and taking into account Remak 4.2, we can express $p_{s, t}$ from the number of the different maximal chains in terms of $(\emptyset, N)$ to $(N, \emptyset)$ which contain $(S, T)$ and $(S \cup\{i\}, T)$ and $q_{s, t}$ in terms of the number of the different chains from $(\emptyset, N)$ to $(N, \emptyset)$ which contain $(S, T \cup\{i\})$ and $(S, T)$.

The following properties propose, in the spirit of the Shapley value, that necessary defender players get a weighted mean worth over all coalitions $(S, T) \in 3^{N}$ and, on the contrary, necessary detractor players pay for a weighted mean worth over all coalitions $(S, T) \in 3^{N}$. The weights in both cases are defined taking into account that (a) all sequential orders of chains have the same probability and (b) all the coalitions of a common size have the same probability of being joined and left.

Definition 4.4 A value $g$ on $\mathcal{B G}_{N}$ satisfies the weighted property of necessary defender player if for all $b \in \mathcal{B G}_{N}$ and for all necessary defender player $i \in N$, it holds that

$$
g_{i}[b]=\frac{2^{n}}{(2 n) !} \sum_{(S, T) \in 3^{N}} \frac{(n+s-t-1) !(n+t-s) !}{2^{s+t-1}} b(S, T) .
$$

Remark 4.5 Notice that, taking into account Remark 4.2, the weights

$$
\frac{(n+s-t-1) !(n+t-s) !}{2^{s+t-1}}
$$

are the number of maximal chains from $(\emptyset, N)$ to $(N, \emptyset)$ which contain $(S \backslash\{i\}, T)$ and $(S, T)$. That is,

$$
c[(\emptyset, N),(S \backslash\{i\}, T)] \cdot c[(S, T),(N, \emptyset)]
$$

Moreover,

$$
\frac{2^{n}}{(2 n) !} \frac{(n+s-t-1) !(n+t-s) !}{2^{s+t-1}}
$$

can be interpreted as the probability that player $i$ joins $S \backslash\{i\}$ in presence of the players in $T$.

Definition 4.6 A value $g$ on $\mathcal{B G}_{N}$ satisfies the weighted property of necessary detractor player if for all $b \in \mathcal{B G}_{N}$ and for all necessary detractor player $i \in N$, it holds that

$$
g_{i}[b]=-\frac{2^{n}}{(2 n) !} \sum_{(S, T) \in 3^{N}} \frac{(n+t-s-1) !(n+s-t) !}{2^{s+t-1}} b(S, T) .
$$

Remark 4.7 From Remark 4.2, it follows that the weights

$$
\frac{(n+t-s-1) !(n+s-t) !}{2^{s+t-1}}
$$


are the number of maximal chains from $(\emptyset, N)$ to $(N, \emptyset)$ which contain $(S, T)$ and $(S, T \backslash\{i\})$.

That is,

$$
c[(\emptyset, N),(S, T)] \cdot c[(S, T \backslash\{i\}),(N, \emptyset)]
$$

Moreover,

$$
\frac{2^{n}}{(2 n) !} \frac{(n+t-s-1) !(n+s-t) !}{2^{s+t-1}}
$$

can be interpreted as the probability that player $i$ leaves $T$ in presence of the players in $S$.

Theorem 4.8 The Shapley bisemivalue is the only value on $\mathcal{B G}_{N}$ that satisfies efficiency, linearity, symmetry, the weighted property of necessary defender player and the weighted property of necessary detractor player.

Proof (Existence) It is straightforward that the Shapley bisemivalue satisfies efficiency, linearity and symmetry.

If $i \in N$ is a necessary defender player in $b$ then,

$$
\begin{aligned}
& \frac{2^{n}}{(2 n) !} \sum_{(S, T) \in 3^{N}} \frac{(n+s-t-1) !(n+t-s) !}{2^{s+t-1}} b(S, T)=\frac{2^{n}}{(2 n) !} \sum_{(S, T) \in 3^{N \backslash\{i\}}}\left[\frac{(n+s-t-1) !(n+t-s) !}{2^{s+t-1}} b(S, T)+\right. \\
& \left.\frac{(n+s-t) !(n+t-s-1) !}{2^{s+t}} b(S \cup\{i\}, T)\right]=\frac{2^{n}}{(2 n) !} \sum_{(S, T) \in 3^{N} \backslash\{i\}} \frac{(n+s-t) !(n+t-s-1) !}{2^{s+t}} b(S \cup\{i\}, T)
\end{aligned}
$$

and then,

$$
\begin{aligned}
\varphi_{i}[b]= & \sum_{(S, T) \in 3^{N \backslash\{i\}}} \frac{(n+s-t) !(n+t-s-1) !}{(2 n) !} 2^{n-s-t}[b(S \cup\{i\}, T)-b(S, T)]+ \\
& \frac{(n+t-s) !(n+s-t-1) !}{(2 n) !} 2^{n-s-t}[b(S, T)-b(S, T \cup\{i\})] \\
= & \sum_{(S, T) \in 3^{N \backslash\{i\}}} \frac{(n+s-t) !(n+t-s-1) !}{(2 n) !} 2^{n-s-t} b(S \cup\{i\}, T) \\
= & \frac{2^{n}}{(2 n) !} \sum_{(S, T) \in 3^{N}} \frac{(n+s-t-1) !(n+t-s) !}{2^{s+t-1}} b(S, T) .
\end{aligned}
$$

Thus, $\varphi$ satisfies the weighted property of necessary defender player.

Moreover, if $i$ is a necessary detractor player, we have that

$$
\begin{aligned}
& -\frac{2^{n}}{(2 n) !} \sum_{(S, T) \in 3^{N}} \frac{(n+t-s-1) !(n+s-t) !}{2^{s+t-1}} b(S, T)=-\frac{2^{n}}{(2 n) !} \sum_{(S, T) \in 3^{N \backslash\{i\}}}\left[\frac{(n+t-s-1) !(n+s-t) !}{2^{s+t-1}} b(S, T)+\right. \\
& \left.\frac{(n+t-s) !(n+s-t-1) !}{2^{s+t}} b(S, T \cup\{i\})\right]=-\frac{2^{n}}{(2 n) !} \sum_{(S, T) \in 3^{N \backslash\{i\}}} \frac{(n+t-s) !(n+s-t-1) !}{2^{s+t}} b(S, T \cup\{i\})
\end{aligned}
$$


and then,

$$
\begin{aligned}
\varphi_{i}[b]= & \sum_{(S, T) \in 3^{N \backslash\{i\}}} \frac{(n+s-t) !(n+t-s-1) !}{(2 n) !} 2^{n-s-t}[b(S \cup\{i\}, T)-b(S, T)]+ \\
& \frac{(n+t-s) !(n+s-t-1) !}{(2 n) !} 2^{n-s-t}[b(S, T)-b(S, T \cup\{i\})] \\
= & -\sum_{(S, T) \in 3^{N \backslash\{i\}}} \frac{(n+t-s) !(n+s-t-1) !}{(2 n) !} 2^{n-s-t} b(S, T \cup\{i\}) \\
= & -\frac{2^{n}}{(2 n) !} \sum_{(S, T) \in 3^{N}} \frac{(n+t-s-1) !(n+s-t) !}{2^{s+t-1}} b(S, T) .
\end{aligned}
$$

Thus, $\varphi$ satisfies the weighted property of necessary detractor player.

(Uniqueness) Let $g$ be a value on $\mathcal{B} \mathcal{G}_{N}$ that satisfies the started properties. We will show that $g$ is uniquely determined on all $b \in \mathcal{B} \mathcal{G}_{N}$; so that it must coincide with $\varphi$. Using linearity, we need only to prove that $g$ is uniquely determined on each identity game $\delta_{(C, D)}$.

Given $(C, D) \in 3^{N}$ such that $(C, D) \neq(\emptyset, \emptyset)$, with $c=|C|$ and $d=|D|$, we will distinguish 3 cases.

(i) If $i \in C, i$ is a necessary defender player in $\delta_{(C, D)}$. By applying the weighted property of necessary defender player and comparing this result with the allocations given in Lemma 2.4 for the particulat case of the Shapley bisemivalue, we get:

$$
\begin{aligned}
g_{i}\left[\boldsymbol{\delta}_{(C, D)}\right] & =\frac{2^{n}}{(2 n) !} \sum_{(S, T) \in 3^{N}} \frac{(n+s-t-1) !(n+t-s) !}{2^{s+t-1}} \delta_{(C, D)}(S, T) \\
& =\frac{2^{n}}{(2 n) !} \frac{(n+c-d-1) !(n+d-c) !}{2^{c+d-1}}=\varphi_{i}\left[\boldsymbol{\delta}_{(C, D)}\right] .
\end{aligned}
$$

(ii) If $i \in D, i$ is a necessary detractor player in $\delta_{(C, D)}$. By applying the weighted property of necessary detractor player and comparing again this result with the allocations given in Lemma 2.4 for the particulat case of the Shapley bisemivalue, we obtain:

$$
\begin{aligned}
g_{i}\left[\boldsymbol{\delta}_{(C, D)}\right] & =-\frac{2^{n}}{(2 n) !} \sum_{(S, T) \in 3^{N}} \frac{(n+t-s-1) !(n+s-t) !}{2^{s+t-1}} \delta_{(C, D)}(S, T) \\
& =-\frac{2^{n}}{(2 n) !} \frac{(n+d-c-1) !(n+c-d) !}{2^{c+d-1}}=\varphi_{i}\left[\boldsymbol{\delta}_{(C, D)}\right] .
\end{aligned}
$$

(iii) If $i \in N \backslash(C \cup D)$, by efficiency, it follows that

$$
\boldsymbol{\delta}_{(C, D)}(N, \emptyset)-\boldsymbol{\delta}_{(C, D)}(\emptyset, N)=\sum_{i \in N} g_{i}\left[\boldsymbol{\delta}_{(C, D)}\right]=\sum_{i \in C} g_{i}\left[\boldsymbol{\delta}_{(C, D)}\right]+\sum_{j \in D} g_{j}\left[\boldsymbol{\delta}_{(C, D)}\right]+\sum_{k \in N \backslash(C \cup D)} g_{k}\left[\boldsymbol{\delta}_{(C, D)}\right] .
$$


By symmetry,

$$
\begin{gathered}
\sum_{i \in C} g_{i}\left[\boldsymbol{\delta}_{(C, D)}\right]=c g_{i}\left[\boldsymbol{\delta}_{(C, D)}\right]=c \varphi_{i}\left[\boldsymbol{\delta}_{(C, D)}\right], \text { for all } i \in C \\
\sum_{j \in D} g_{j}\left[\boldsymbol{\delta}_{(C, D)}\right]=d g_{j}\left[\boldsymbol{\delta}_{(C, D)}\right]=d \varphi_{j}\left[\boldsymbol{\delta}_{(C, D)}\right], \text { for all } j \in D, \text { and } \\
\sum_{k \in N \backslash(C \cup D)} g_{k}\left[\boldsymbol{\delta}_{(C, D)}\right]=(n-c-d) g_{k}\left[\boldsymbol{\delta}_{(C, D)}\right], \text { for all } k \in N \backslash(C \cup D) .
\end{gathered}
$$

Finally, we can deduce

$$
g_{i}\left[\boldsymbol{\delta}_{(C, D)}\right]=\varphi_{i}\left[\boldsymbol{\delta}_{(C, D)}\right], \text { for all } i \in N \backslash(C \cup D)
$$

Proposition 4.9 (Logical independence of the system used in Theorem 4.8) We will assume that a player set $N$ (with $n=|N| \geq 2$ ) is given.

We will abbreviate the properties as follows: $E F=$ efficiency, $L I=$ linearity, $S Y=$ symmetry property, $W D E F=$ necessary defender player weighted property and WDET = necessary detractor player weighted property.

\section{EF is logically independent of LI, SY, WDEF and WDET}

We define the value $g \in \mathcal{B G}_{N}$ as follows:

(a) If $n=2$ then,

$$
\begin{aligned}
& g_{1}[b]=\varphi_{1}[b]-[b(\{2\}, \emptyset)-b(\emptyset,\{2\})] \\
& g_{2}[b]=\varphi_{2}[b]-[b(\{1\}, \emptyset)-b(\emptyset,\{1\})] .
\end{aligned}
$$

(b) Otherwise, that is, if $n>2$, we define the value $g_{i}[b]=\varphi_{i}[b]$, for all $i \in N$.

This value satisfies LI, SY, WDEF and WDET. However, it fails to satisfy EF for $n=2$.

\section{LI is logically independent of EF, SY, WDEF and WDET}

We define the value $g$ on $\mathcal{B G}_{N}$ as follows:

(a) Identity games. If $b=\delta_{(A, B)}$ with $(A, B) \neq(\emptyset, \emptyset)$ then,

$$
g_{i}[b]=\varphi_{i}[b]
$$

(b) Otherwise, that is, if $b$ is not a identity game, then, we will denote by $D_{1}$ the set of necessary defender players and $D_{2}$ the set of necessary detractor players in $b$. We define the value $g$ on $\mathcal{B} \mathcal{G}_{N}$ as follows:

$$
g_{i}[b]= \begin{cases}\varphi_{i}[b] & \text { if } i \in D_{1} \cup D_{2} \\ \frac{1}{n-\left(\left|D_{1}\right|+\left|D_{2}\right|\right)}\left[b(N, \emptyset)-b(\emptyset, N)-\left(\sum_{j \in D_{1}} \varphi_{j}[b]+\sum_{k \in D_{2}} \varphi_{k}[b]\right)\right] & \text { if } i \in N \backslash\left(D_{1} \cup D_{2}\right) .\end{cases}
$$


This value satisfies EF, SY, WDEF and DET. However, it fails to satisfy LI for e.g. game $b=\delta_{(1, \emptyset)}+2 \delta_{(\emptyset, 2)}$ and $n=2$.

3. SY logically independent of EF, LI, WDEF and WDET.

We define the value $g \in \mathcal{B} \mathcal{G}_{N}$ as follows:

(a) If $n=3$, then,

$$
\begin{aligned}
& g_{1}[b]=\varphi_{1}[b]-[b(\{2\}, \emptyset)-b(\emptyset,\{2\})-b(\{3\}, \emptyset)+b(\emptyset,\{3\})], \\
& g_{2}[b]=\varphi_{2}[b]+[b(\{1\}, \emptyset)-b(\emptyset,\{1\})-b(\{3\}, \emptyset)+b(\emptyset,\{3\})], \\
& g_{3}[b]=\varphi_{3}[b]-[b(\{1\}, \emptyset)-b(\emptyset,\{1\})-b(\{2\}, \emptyset)+b(\emptyset,\{2\})] .
\end{aligned}
$$

(b) Otherwise, that is, if $n \neq 3$, we define the value $g_{i}[b]=\varphi_{i}[b]$, for all $i \in N$.

This value satisfies EF, LI, DEF and DET. However, it fails to satisfy SY.

4. WDEF is logically independent of EF, LI, SY, and WDET.

We define the value $g \in \mathcal{B G}_{N}$ as follows:

(a) If $n=2$ then,

$$
\begin{aligned}
& g_{1}[b]=\varphi_{1}[b]+[b(\{1\}, \emptyset)-b(\{2\}, \emptyset)], \\
& g_{2}[b]=\varphi_{2}[b]-[b(\{1\}, \emptyset)-b(\{2\}, \emptyset)] .
\end{aligned}
$$

(b) Otherwise, that is, if $n>2$, we define the value $g_{i}[b]=\varphi_{i}[b]$, for all $i \in N$.

This value satisfies EF, LI, SY and WDET. However, it fails to satisfy WDEF.

5. WDET is logically independent of EF, LI, SY and WDEF.

We define the value $g \in \mathcal{B G}_{N}$ as follows:

(a) If $n=2$ then,

$$
\begin{aligned}
& g_{1}[b]=\varphi_{1}[b]+[b(\emptyset,\{1\})-b(\emptyset,\{2\})], \\
& g_{2}[b]=\varphi_{2}[b]-[b(\emptyset,\{1\})-b(\emptyset,\{2\})] .
\end{aligned}
$$

(b) Otherwise, that is, if $n>2$, we define the value $g_{i}[b]=\varphi_{i}[b]$, for all $i \in N$.

This value satisfies EF, LI, SY, and WDEF. However, it fails to satisfy WDET.

\section{A computational procedure}

As it is well known, both the Shapley and Banzhaf values of any cooperative game can be easily obtained from its multilinear extension. Indeed, as it is proved in [29], the Shapley value can be calculated by integrating the partial derivatives of the multilinear extension of the game along the main diagonal $x_{1}=x_{2}=\cdots=x_{n}$ of the cube $[0,1]^{n}$, while the partial derivatives 
of that multilinear extension evaluated at point $(1 / 2,1 / 2, \ldots, 1 / 2)$ give the Banzhaf value (see [30]). In [24], this latter procedure is extended to any multinomial probabilistic value and in [11], semivalues on cooperative games are also computed by means of the multilinear extension of the game.

Following this idea, Domènech et al [16] defined the multilinear extension of a bicooperative game (MLE), that parallels the existing definition of the multilinear extension of a cooperative game given by Owen, and provided a method to compute $(p, q)$ - bisemivalues in general, and the Banzhaf bisemivalue in particular, by means of it. In a recent paper, Domènech et al [17] go further and give a computational procedure to calculate any bisemivalue, also by using the MLE of the game defined in [16].

In this section, we introduce a new representation of the multilinear extension of a bicooperative game and provide a procedure to compute the Shapley bisemivalue by means of it.

First of all, we identify each $(S, T) \in 3^{N}$ by vectors $(X, Y, Z)$ of $\mathbb{R}^{3 n}$ such that $X=\left(x_{1}, \ldots, x_{n}\right)$, $Y=\left(y_{1}, \ldots, y_{n}\right), Z=\left(z_{1}, \ldots, z_{n}\right)$ and

$$
x_{i}=\left\{\begin{array}{ll}
1 & \text { if } i \in S \\
0 & \text { otherwise }
\end{array}, \quad y_{i}=\left\{\begin{array}{ll}
1 & \text { if } i \in T \\
0 & \text { otherwise }
\end{array} \text { and } \quad z_{i}= \begin{cases}1 & \text { if } i \in N \backslash(S \cup T) \\
0 & \text { otherwise }\end{cases}\right.\right.
$$

For instead, if $N=\{1,2,3\}$ the coalitions $(\{1,3\},\{2\})$ and $(\{1,2\}, \emptyset)$ are identified by $(X, Y, Z)=(1,0,1,0,1,0,0,0,0)$ and $(X, Y, Z)=(1,1,0,0,0,0,0,0,1)$ respectively.

Definition 5.1 The generalized multilinear extension of a game $b \in \mathcal{B G}_{N}$ is the real-valued function defined on $\mathbb{R}^{3 n}$ by

$$
f(X, Y, Z)=\sum_{(S, T) \in 3^{N}}\left[\prod_{i \in S} x_{i} \prod_{j \in T} y_{j} \prod_{k \in N \backslash(S \cup T)} z_{k}\right] b(S, T) .
$$

where $X=\left(x_{1}, \ldots, x_{n}\right), Y=\left(y_{1}, \ldots, y_{n}\right), Z=\left(z_{1}, \ldots, z_{n}\right) \in[0,1]^{n}$.

It is easy to prove that $f$ coincides with $b$ where $b$ is defined.

Lemma 5.2 The weighting coefficients $p_{s, t}, q_{s, t}, s, t=0, \ldots, n-1$, associated to the Shapley value, and defined in (4), can be calculated as

$$
\begin{aligned}
& p_{s, t}=2^{n} \int_{0}^{1} p^{n}(1-p)^{n-1}\left(\frac{p}{2(1-p)}\right)^{s}\left(\frac{1-p}{2 p}\right)^{t} d p \\
& q_{s, t}=2^{n} \int_{0}^{1} p^{n}(1-p)^{n-1}\left(\frac{1-p}{2 p}\right)^{s}\left(\frac{p}{2(1-p)}\right)^{t} d p .
\end{aligned}
$$

Proof Taking into account that

$$
\beta(a, b)=\int_{0}^{1} x^{a-1}(1-x)^{b-1} d x=\frac{\Gamma(a) \Gamma(b)}{\Gamma(a+b)}
$$

and

$$
\Gamma(a)=(a-1) !, \text { if } a \in \mathbb{N},
$$


we can write

$$
\begin{aligned}
p_{s, t} & =\frac{(n+s-t) !(n+t-s-1) !}{(2 n) !} 2^{n-s-t}=2^{n-s-t} \frac{\Gamma(n+s-t+1) \Gamma(n+t-s)}{\Gamma(2 n+1)} \\
& =2^{n-s-t} \beta(n+s-t+1, n+t-s)=2^{n-s-t} \int_{0}^{1} p^{n+s-t}(1-p)^{n+t-s-1} d p \\
& =2^{n} \int_{0}^{1} p^{n}(1-p)^{n-1}\left(\frac{p}{2(1-p)}\right)^{s}\left(\frac{1-p}{2 p}\right)^{t} d p
\end{aligned}
$$

Analogously, for $q_{s, t}$ we have

$$
\begin{aligned}
q_{s, t} & =\frac{(n+t-s) !(n+s-t-1) !}{(2 n) !} 2^{n-s-t}=2^{n-s-t} \frac{\Gamma(n+t-s+1) \Gamma(n+s-t)}{\Gamma(2 n+1)} \\
& =2^{n-s-t} \beta(n+t-s+1, n+s-t)=2^{n-s-t} \int_{0}^{1} p^{n+t-s}(1-p)^{n+s-t-1} d p \\
& =2^{n} \int_{0}^{1} p^{n}(1-p)^{n-1}\left(\frac{1-p}{2 p}\right)^{s}\left(\frac{p}{2(1-p)}\right)^{t} d p
\end{aligned}
$$

Proposition 5.3 Let $\varphi$ be the Shapley value and $f$ is the generalized multilinear extension of a game $b \in \mathcal{B G}_{N}$. Then, for all $i \in N$

$$
\begin{aligned}
& \varphi_{i}[b]=2^{n} \int_{0}^{1} p^{n}(1-p)^{n-1}\left[\left(\frac{\partial f}{\partial x_{i}}-\frac{\partial f}{\partial z_{i}}\right)\left(\frac{P}{2(1-P)}, \frac{1-P}{2 P}, \mathbf{1}\right)+\left(\frac{\partial f}{\partial z_{i}}-\frac{\partial f}{\partial y_{i}}\right)\left(\frac{1-P}{2 P}, \frac{P}{2(1-P)}, \mathbf{1}\right)\right] d p, \\
& \text { where } \frac{P}{2(1-P)}=\left(\frac{p}{2(1-p)}, \stackrel{n}{\cdots}, \frac{p}{2(1-p)}\right), \frac{1-P}{2 P}=\left(\frac{1-p}{2 p}, \stackrel{n}{.}, \frac{1-p}{2 p}\right) \text { and } \mathbf{1}=(1, \stackrel{n}{.}, 1) .
\end{aligned}
$$

Proof From Definition 5.1, the partial derivatives of $f$ with respect to $x_{i}, y_{i}, z_{i}$ are:

$$
\begin{aligned}
& \frac{\partial f}{\partial x_{i}}(X, Y, Z)=\sum_{(S, T) \in 3^{N \backslash i}}\left[\prod_{j \in S} x_{j} \prod_{k \in T} y_{k} \prod_{l \in N \backslash(S \cup T \cup i)} z_{l}\right] b(S \cup i, T), \\
& \frac{\partial f}{\partial y_{i}}(X, Y, Z)=\sum_{(S, T) \in 3^{N \backslash i}}\left[\prod_{j \in S} x_{j} \prod_{k \in T} y_{k} \prod_{l \in N \backslash(S \cup T \cup i)} z_{l}\right] b(S, T \cup i), \\
& \frac{\partial f}{\partial z_{i}}(X, Y, Z)=\sum_{(S, T) \in 3^{N \backslash i}}\left[\prod_{j \in S} x_{j} \prod_{k \in T} y_{k} \prod_{l \in N \backslash(S \cup T \cup i)} z_{l}\right] b(S, T),
\end{aligned}
$$

then

$$
\begin{aligned}
& \frac{\partial f}{\partial x_{i}}(X, Y, Z)-\frac{\partial f}{\partial z_{i}}(X, Y, Z)=\sum_{(S, T) \in 3^{N \backslash i}}\left[\prod_{j \in S} x_{j} \prod_{k \in T} y_{k} \prod_{l \in N \backslash(S \cup T \cup i)} z_{l}\right][b(S \cup i, T)-b(S, T)] \text { (6) } \\
& \frac{\partial f}{\partial z_{i}}(X, Y, Z)-\frac{\partial f}{\partial y_{i}}(X, Y, Z)=\sum_{(S, T) \in 3^{N \backslash i}}\left[\prod_{j \in S} x_{j} \prod_{k \in T} y_{k} \prod_{l \in N \backslash(S \cup T \cup i)} z_{l}\right][b(S, T)-b(S, T \cup i)] \text { (7) }
\end{aligned}
$$


Finally, valuating (6) at point $\left(\frac{P}{2(1-P)}, \frac{1-P}{2 P}, \mathbf{1}\right)$ and (7) at point $\left(\frac{1-P}{2 P}, \frac{P}{2(1-P)}, \mathbf{1}\right)$,

adding these two results, multiplying by $2^{n} p^{n}(1-p)^{n-1}$, integrating between 0 and 1 and by using Lemma 5.2, we obtain the Shapley value

$$
\varphi_{i}[b]=\sum_{(S, T) \in 3^{N \backslash i}} p_{s, t}[b(S \cup i, T)-b(S, T)]+q_{s, t}[b(S, T)-b(S, T \cup i)] .
$$

Example 5.4 Two car manufacturers, $A_{1}$ and $A_{2}$, are always in competition in order to obtain the maximum number of sales in a market. If $N$ represents the set of component suppliers of these two companies, we can define the bicooperative game $b(S, T)$ as $A_{1}$ 's profit when players in $S$ work for $A_{1}$, players in $T$ work for $A_{2}$ and players in $N \backslash(S \cup T)$ do not work for neither $A_{1}$ nor $A_{2}$.

Consider $N=\{1,2,3\}$ the set of component suppliers and assume that suppliers 1 and 3 are those that provide the maximum and minimum profit margin to companies respectively. If a supplier stops providing parts to manufacturer $A_{1}$, he/she can provide them to $A_{2}$ or, on the contrary, start working to another type of company unrelated to the automotive sector, to end its business, ... In the first case, company $A_{1}$ will be more damaged rather than in the second one.

In this situation, let $b$ be the bicooperative game defined by

$$
\begin{array}{lll}
b(\{1,2,3\}, \emptyset)=100, & b(\emptyset, \emptyset)=0, & b(\emptyset,\{1,2,3\})=-60, \\
b(\{1,3\}, \emptyset)=85, & b(\{2,3\}, \emptyset)=75, & b(\{1,2\}, \emptyset)=90, \\
b(\{1,3\},\{2\})=50, & b(\{2,3\},\{1\})=20, & b(\{1,2\},\{3\})=60, \\
b(\{3\}, \emptyset)=65, & b(\{2\}, \emptyset)=70, & b(\{1\}, \emptyset)=80, \\
b(\{3\},\{1\})=5, & b(\{3\},\{2\})=15, & b(\{2\},\{1\})=10, \\
b(\{2\},\{3\})=35, & b(\{1\},\{2\})=40, & b(\{1\},\{3\})=50, \\
b(\{3\},\{1,2\})=-25, & b(\{2\},\{1,3\})=-20, & b(\{1\},\{2,3\})=5, \\
b(\emptyset,\{1\})=-30, & b(\emptyset,\{2\})=-15, & b(\emptyset,\{3\})=-10, \\
b(\emptyset,\{2,3\})=-30, & b(\emptyset,\{1,3\})=-40, & b(\emptyset,\{1,2\})=-50
\end{array}
$$

From Definition 5.1, the generalized multilinear extension of $b$ is

$$
\begin{aligned}
f(X, Y, Z)= & 100 x_{1} x_{2} x_{3}+85 x_{1} x_{3} z_{2}+50 x_{1} x_{3} y_{2}+65 x_{3} z_{1} z_{2}+5 x_{3} y_{1} z_{2}+35 x_{2} y_{3} z_{1}- \\
& 25 x_{3} y_{1} y_{2}-30 y_{1} z_{2} z_{3}-30 y_{2} y_{3} z_{1}+75 x_{2} x_{3} z_{1}+20 x_{2} x_{3} y_{1}+70 x_{2} z_{1} z_{3}+ \\
& 15 x_{3} y_{2} z_{1}+40 x_{1} y_{2} z_{3}-20 x_{2} y_{1} y_{3}-15 y_{2} z_{1} z_{3}-40 y_{1} y_{3} z_{2}-60 y_{1} y_{2} y_{3}+ \\
& 90 x_{1} x_{2} z_{3}+60 x_{1} x_{2} y_{3}+80 x_{1} z_{2} z_{3}+10 x_{2} y_{1} z_{3}+50 x_{1} y_{3} z_{2}+5 x_{1} y_{2} y_{3}- \\
& 10 y_{3} z_{1} z_{2}-50 y_{1} y_{2} z_{3}
\end{aligned}
$$

We compute the Shapley bisemivalue using the multilinear extension technique given in 
Proposition 5.3 as follows:

$$
\begin{aligned}
& \varphi_{1}[b]=2^{3} \int_{0}^{1} p^{3}(1-p)^{2}\left[\frac{5}{4} \frac{12 p^{4}+14 p^{3}-55 p^{2}+22 p+18}{p^{2}(p-1)^{2}}\right] d p=73.83 \\
& \varphi_{2}[b]=2^{3} \int_{0}^{1} p^{3}(1-p)^{2}\left[\frac{5}{4} \frac{12 p^{4}+14 p^{3}-56 p^{2}+26 p+11}{p^{2}(p-1)^{2}}\right] d p=49.67 \\
& \varphi_{3}[b]=2^{3} \int_{0}^{1} p^{3}(1-p)^{2}\left[\frac{5}{4} \frac{26 p^{4}-28 p^{3}-13 p^{2}+8 p+11}{p^{2}(p-1)^{2}}\right] d p=36.50 .
\end{aligned}
$$

Notice that

$$
\sum_{i \in N} \varphi_{i}[b]=b(N, \emptyset)-b(\emptyset, N)=160
$$

\section{Conclusions}

In this paper we characterize the Banzhaf and the Shapley bisemivalues by introducing new non-standard properties related to three special kind of players: weak null, necessary defender and necessary detractor players. In the case of a null player, all contributions vanish, but it is not true for the case of weak null players. In general, a necessary player is one such that all pair of coalitions $(S, T)$ not containing him/her have zero worth. In particular, we have distinguish two cases depending on the fact that the player does not belong to $S$ - necessary defender player- or does not belong to $T$ - necessary detractor player-. There are several possibilities to allocate payoffs to these players and in the three axiomatic characterizations, given in Theorem 3.9, Theorem 3.11 and Theorem 4.8, we have tried to allocate reasonable payoffs to each one of them.

It is important to highlight that these new axiomatic characterizations of the Banzhaf bisemivalue on the set of all bicooperative games (with only three properties, instead of the five properties used in [9] when characterize the Banzhaf power index on the set of ternary bicooperative games) does not include total power property and replaces this property and the structural property, given in [9], with the more intuitive properties of necessary defender player (Theorem 3.9) and necessary detractor player (Theorem 3.11). In the case of the Shapley bisemivalue, the axiomatic characterization (with five properties, as in [6]), is the result of removing the structural and the dummy player properties given in [6] and, as in the case of the Banzhaf bisemivalue, introducing two more intuitive properties: the weighted properties of necessary defender and necessary detractor player, making use of a weighted mean.

Finally, we provide a computational procedure to calculate the allocations given by the Shapley bisemivalue by introducing the generalized multilinear extension of a bicooperative game.

\section{Acknowledgments}

The authors wish to thank the managing editor for encouraging them to improve the paper, and two anonymous reviewers for their interesting comments and helpful suggestions, most of which have been incorporated into the text. 


\section{References}

[1] Algaba, E., Fragnelli, V. and Sánchez-Soriano, J. [2019]: Handbook of the Shapley Value, CRC Press, Taylor \& Francis, USA.

[2] Alonso-Meijide, J.M., Costa, J. and Garcia Jurado, I. [2019]: "Values, Nullifiers and Dummifiers." In: Handbook of the Shapley Value, 75-92.

[3] Alonso-Meijide, J.M., Costa, J. and Garcia Jurado, I. [2019]: "Null, nullifying and necessary agents: parallel characterizations of the Banzhaf and Shapley values." Journal of Optimization Theory and Applications 180, 1027-1035.

[4] Banzhaf, J.F. [1965]: "Weigthed voting doesn't work: A mathematical analysis." Rutgers Law Review 19, 317-343.

[5] Bilbao, J.M. [2000]: "Bicooperative games." Cooperation games on combinatorial structures. Kluwer Academic Publishers.

[6] Bilbao, J.M., Fernández, J.R., Jiménez, N. and López, J.J. [2000]: “The Shapley value for bicooperative games" Annals of Operations Research 158, 99-115.

[7] Bilbao, J.M., Fernández, J.R., Jiménez, N. and López, J.J. [2007]: "The core and the Weber set for bicooperative games" International Journal of Game Theory 36, 209-222.

[8] Bilbao, J.M., Fernández, J.R., Jiménez, N. and López, J.J. [2008]: "Biprobabilistic values for bicooperative games" Discrete Applied Mathematics 156, 2698-2711.

[9] Bilbao, J.M., Fernández, J.R., Jiménez, N. and López, J.J. [2010]: “The Banzhaf power index for ternary bicooperative games" Discrete Applied Mathematics 158, 967-980.

[10] Carreras, F., Freixas, J. and Puente, M.A. [2003]: "Semivalues as power indices." European Journal of Operational Research 149, 676-687.

[11] Carreras, F., Giménez, J.M. [2011]: Power and potential maps induced by any semivalue: Some algrebraic properties and computation by multilinear extension. European Journal of Operational Research 211, 148-159.

[12] Casajus, A., Huettner, F. [2014]: "Null, nullifying or dummifying players: The difference between the Shapley value, the equal division value and the equal surplus value." Economics Letters 122, 167-169.

[13] Coleman, J.S. [1971]: "Control of collectivities and the power of a collectivity to act." In: Social Choice (B. Lieberman, ed.), Gordon and Breach, New York, 269-300.

[14] Deegal, J., Packel, E.W. [1978]: "A new index of power for simple $n$-personal games". International Journal of Game Theory 7, 113-123.

[15] Domènech, M., Giménez, J.M. and Puente, M.A. [2016]: "Some properties for probabilistic and multinomial (probabilistic) values on cooperative games." Optimization 65, $1377-1395$. 
[16] Domènech M., Giménez, J.M and Puente, M.A. 1[2018]: "Bisemivalues for bicooperative games". Optimization 67:6, 907-919.

[17] Domènech M., Giménez, J.M and Puente, M.A. [2020]: "Some properties for bisemivalues on bicooperative games." Journal of Optimization Theory and Applications 185, 270-288.

[18] Dubey, P. [1975]: “On the uniqueness of the Shapley value.” International Journal of Game Theory 4, 131-139.

[19] Dubey, P., Neyman, A. and Weber, R.J. [1981]: "Value theory without efficiency." Mathematics of Operations Research 6, 122-128.

[20] Feltkamp, V. [1995]: "Alternative axiomatic characterizations of the Shapley and Banzhaf values." International Journal of Game Theory 24, 179-186.

[21] Felsenthal, D. and Machover, M. [1997]: “Ternary voting games” International Journal of Game Theory 26, 335-351.

[22] Freixas, J. [2005]. "The Shapley-Shubik power index for games with several levels of approval in the input and output." Decision Support Systems 39, 185-195.

[23] Freixas, J. [2005]. "Banzhaf measures for games with several levels of approval in the input and output." Annals of Operations Research 137, 45-66.

[24] Freixas, J. and Puente, M.A. [2002]: "Reliability importance measures of the components in a system based on semivalues and probabilistic values." Annals of Operations Research 109, 331-342.

[25] Freixas, J. and Zwicker, W.S. [2003]. "Weighted voting, abstention, and multiple levels of approval." Social Choice and Welfare 21, 399-431.

[26] Grabisch, M. and Labreuche, Ch. [2005]; "Bi-capacities-I: Definition, Möbius transform and interaction", Fuzzy Sets and Systems 151, 211-236.

[27] Laruelle, A. and Valenciano, F. [2001a]: "Shapley-Shubik and Banzhaf indices revisited." Mathematics of Operations Research 26, 89-104.

[28] Lehrer, E. [1988]: “An axiomatization of the Banzhaf value.” International Journal of Game Theory 17, 89-99.

[29] Owen, G. [1972]: “Multilinear extensions of games.” Management Science 18, 64-79.

[30] Owen, G. [1975]: "Multilinear extensions and the Banzhaf value." Naval Research Logistics Quarterly 22, 741-750.

[31] Owen, G. [1978]: "Characterization of the Banzhaf-Coleman index." SIAM Journal of Applied Mathematics 35, 315-327.

[32] Penrose, L.S. [1946]: “The elementary statistics of majority voting." Journal of the Royal Statistical Society 109, 53-57. 
[33] Shapley, L.S. [1953]: “A value for n-person games.” In: Contributions to the Theory of Games II (H.W. Kuhn and A.W. Tucker, eds.), Princeton University Press, 307-317.

[34] Shapley, L.S. and Shubik, M. [1954]: "A method for evaluating the distribution of power in a committee system." American Political Science Review 48, 787-792.

[35] Van den Brink, R. [2007]: "Null or nullifying players: the difference between the Shapley value and equal division solutions." Journal of Economic Theory 136, 767-775. 